\title{
Hebbian Learning for Olfactory Sequences
}

\author{
Andrew J. Johnson ${ }^{1}$, Laura Cauchi ${ }^{2}$, and Christopher Miles ${ }^{3}$ \\ 1Psychology Research Centre \\ Bournemouth University, \\ Poole, \\ United Kingdom
${ }^{2}$ Department of Psychology and
Behavioural Sciences,
Coventry University,
Coventry, United Kingdom
${ }^{3}$ School of Psychology
Cardiff University, Cardiff,
United Kingdom

Corresponding Author:

Address for Correspondence:
Andrew J. Johnson

Psychology Research Centre

Bournemouth University

Fern Barrow

Poole

BH12 5BB

Email: andjohnson@ bournemouth.ac.uk

Telephone: 01202965977

Short title: Odour Hebb Effect 


\begin{abstract}
The present paper explores the generality of the Hebb repetition effect to the learning of olfactory sequences in order to assess commonality of memory functioning across sensory modalities. Participants completed a serial order reconstruction task comprising sequences of four olfactory stimuli. Following presentation of each sequence, participants were represented with the odours and required to reconstruct their order of presentation. Surreptitious representation of the repeated sequence occurred on every third trial. This order reconstruction task produced a serial position function comprising recency only for both the non-repeated and repeated sequences. Importantly, serial order reconstruction for the repeated odour sequence produced improved performance for that sequence compared to the non-repeated sequences. This observation of a Hebb repetition effect for olfactory sequences further supports the proposition that sequential learning can operate amodally (e.g. Guérard \& Tremblay, 2008).
\end{abstract}

\title{
Introduction
}

In a typical Hebbian learning procedure (Hebb, 1961) participants are repeatedly presented with a sequence of items for immediate serial recall. One sequence is surreptitiously repeated on every $3^{\text {rd }}$ trial and is known as the Hebb (or, repeated) sequence. Hebb (1961) showed that, over a number of trials, serial recall for the repeated sequence improved relative to the non-repeated sequence. That is, across an experimental session, Hebbian learning is typically characterised by a learning gradient for the repeated sequence which is steeper than that for the non-repeated sequences. Although Hebbian learning was originally conceptualised as a form of implicit learning (Hebb, 1961), subsequent studies have shown that it is unaffected by participants' conscious awareness of the repeated sequence (Couture \& Tremblay, 2006; see also Szmalec, Loncke, Duyck \& Page, 2011, for discussion).

Hebbian learning has been associated with language acquisition, and conceptualised as an analogue for the learning of novel vocabulary (e.g. Szmalec, Duyck, Vandierendonck, Barberá Mata \& Page, 2009; Mosse and Jarrold, 2008). However, notwithstanding the link with language, Hebb repetition effects have been shown across a range of verbal and nonverbal stimulus types, e.g., unfamiliar-faces (Horton, Hay \& Smyth, 2008), nameable pictures (Page, Cumming, Norris, Hitch \& McNeil, 2006), spatial position of auditory speech sounds (Parmentier, Maybery, Huitson \& Jones, 2008), spatial position of visual stimuli and (Couture \& Tremblay, 2006; Mosse \& Jarrold, 2010). Interestingly, Hebbian learning for the spatial position of white noise bursts was initially reported as absent (Parmentier et al., 2008). However, reanalysis of the same data set (Lafond, Tremblay \& Parmentier, 2010) revealed that this null effect of sequence learning could be attributed to error learning. Additionally, there is evidence that Hebbian learning can transfer between modalities (Page et al., 2006). The reports of Hebbian learning across a range modalities, combined with analogous serial position and error functions (Guérard \& Tremblay, 2008), have encouraged the proposition that sequential learning is represented via an abstract, modality-independent, mechanism. Indeed, Parmentier (2011) proposes an amodal mechanism for order memory in which the pattern of neuronal activity, irrespective of the neurons employed, facilitates the acquisition 
of sequential memory. Such an account (see also Guérard \& Tremblay, 2008) enables the abstract, cross-modal representation of serial order whilst accommodating the observation that neuronal activation for order memory occurs at modality-specific locations (e.g. Smith, Jonides \& Koeppe, 1996). Moreover, the proposition of a universal mechanism for serialorder memory is supported by the qualitatively equivalent serial position functions reported across a range of stimuli (e.g. Guérard \& Tremblay, 2008; Parmentier \& Jones, 2000; Ward, Avons \& Melling, 2005). Such equivalence led Ward et al. (2005) to propose that the serialorder reconstruction task is characterised by both primacy and recency, irrespective of the type of stimuli employed.

The universality of serial order memory functions has, however, been questioned by those studies employing olfactory stimuli. For example, following a single-probe serial-order recall task, Johnson \& Miles (2009) reported qualitatively different serial position functions for olfactory stimuli, compared to those for unfamiliar face and pure-tone stimuli. This qualitative inconsistency was apparent despite evidence suggesting that the serial position function for odour recognition mirrored that for other stimulus types (Johnson \& Miles, 2007; Miles \& Hodder, 2005). The primary aim of the current study is to determine the extent to which Hebbian learning for olfactory sequences is evidenced, despite the serial order recall function for olfactory stimuli differing to that typically observed for unfamiliar face and puretone stimuli.

Previous applications of the Hebb paradigm have comprised either serial recall for verbal material (e.g. Hebb, 1961) or reconstruction of presentation order via the sequential selection of each item from a visual platform (e.g. Horton et al., 2008). Both recall procedures are inappropriate for olfactory stimuli: verbal serial recall necessitates verbal recoding of the odours (see Miles \& Jenkins, 2000), and serial reconstruction necessitates the simultaneous re-presentation of sequence items in order for the participant to identify the order of presentation. Olfactory stimuli cannot be presented simultaneously, thereby precluding such a recall procedure. To combat both limitations particular to olfactory stimuli, a novel serial order recall paradigm is described. Following sequential presentation of the olfactory stimuli at learning, all stimuli are re-presented to the participant at test, and participants are required to reconstruct their order of original presentation via independent evaluation of each odour. To the extent that the novel reconstruction task mirrors the demands of serial order reconstruction (i.e. reassess sequence items at test and indicate the order in which they were originally presented), we predict that the serial position function will mirror the signature characteristics of serial order reconstruction, i.e. primacy and recency (e.g. Ward et al., 2005).

In the present study, we examine the learning for olfactory order memory across a 30-trial experimental session. In order to minimise the opportunity for verbal recoding of the odours, we selected hard-to-name, non-food odours, and these were presented in conjunction with the requirement for inter-stimulus-interval (ISI) articulatory suppression. Differences in neurological activation are evidenced for the learning and recall of nameable and nonnameable odours (Zelano, Montag, Khan \& Sobel, 2009). Specifically, for nameable odours, greater activation is found in the left opercular and orbital inferior frontal gyrus (consistent 
with employment of the phonological loop), whilst for non-nameable odours, greater levels of activity are evident in the primary olfactory cortex (Zelano et al., 2009). These findings have been interpreted as evidence for the existence of an independent olfactory 'module' in working memory (e.g. see Andrade \& Donaldson, 2007). This, in turn, allows the argument that evidence for Hebbian repetition learning for olfactory sequences does not merely reflect the learning of a particular verbal sequence.

\section{Analytic Methodology}

There exists a range of analytical statistical methodologies to test for the Hebb repetition effect (Parmentier et al., 2008) and, because of the novelty of applying olfactory stimuli to this paradigm, we describe and apply two distinct methodologies. First, independent regression analysis for the non-repeated and repeated sequences provides an estimate of incremental learning across epochs. To the extent that Hebbian learning is evident, the gradient of improvement for the repeated sequence should be steeper than that for the nonrepeated sequences. As noted by Parmentier et al. (2008), an advantage of this technique is that differences in the gradients of improvement cannot be attributed to practice per se. This methodology is, however, constrained by its insensitivity to the relative late-onset (in terms of epoch number) of Hebb repetition learning (Parmentier et al., 2008). Therefore, a second approach is described in which participants' recall accuracy for the non-repeated and repeated sequences is compared for the first and last experimental epoch. Again, to the extent that Hebbian learning is evident, this analysis predicts an interaction such that recall difference between the non-repeated and repeated sequences is found at the last epoch only.

\section{Method}

Participants: Twenty-four Coventry University Psychology undergraduates (mean age $=21$ years, 4 months: 6 male and 18 female) participated in exchange for course credit. Ethical approval was obtained from the Coventry University Psychology Ethics Committee.

Materials: The stimuli comprised 10 odour cubes (Dale Air Ltd, UK) selected at random from a corpus of 120 non-food related odours. Each odour was presented as a liquid soaked in gauze contained within a $50 \mathrm{~mm} \times 50 \mathrm{~mm} \times 50 \mathrm{~mm}$ green coloured cube. One face of the cube contained six perforations arranged in a circular array from which the odour was inhaled. To maintain the integrity of individual odours, a protective sticker was placed over the perforations when the odour cube was not being used.

Design: A 2x10x4 within-participants design was employed, where the first factor refers to sequence type (repeated versus non-repeated), the second to experimental epoch (1-10), and the third to serial position (1-4). An experimental epoch comprises 3 sequences: one repeated sequence and its two preceding, non-repeated sequences. The study comprised the presentation of 30 sequences ( 20 non-repeated and 10 repeated) and each sequence comprised 4 odours. For each participant, the same 4 odours were employed throughout the experiment, and these were selected at random for each participant from the set of 10 . 
Procedure: Participants were tested individually in a well-ventilated, quiet laboratory. A pilot study $(n=10)$ established that a 4-odour sequence allowed adequate scope for performance increments following repeated sequence exposure (mean serial position recall for 10 trials $=39.75 \%$ ).

The structure of the Hebbian learning procedure described here is consistent with a range of past studies (e.g. Hebb, 1961; Horton et al., 2008; Page et al., 2006; Parmentier et al. 2008 etc). Each epoch comprised three sequences: two sequences are 'filler' sequences and one sequence is the repeated (Hebb) sequence. The repeated (Hebb) sequence occurs in position 3 of each epoch, and thus each repeated (Hebb) sequence is separated by 2 non-repeated (filler) sequences. For purposes of analysis the average (mean) correct score for each pair of filler sequences is compared with the correct score for the Hebb sequence within each epoch.

The participant faced the experimenter across a table. In order to minimise visual cues for sequence order, each odour was presented over the centre of a screen and held under the nose of the participant for a $3 \mathrm{~s}$ period. The participant was instructed to inhale deeply through both nostrils for the duration of each odour presentation. The odour was then re-placed behind the screen. In order to minimise the possibility of sub-vocal rehearsal, the participant was instructed to repeat the numbers " $1,2,3,4$ " aloud and at a rate of 3-4 digits per second throughout the $3 \mathrm{~s}$ ISI. A $3 \mathrm{~s}$ retention interval followed presentation of the fourth odour during which the odours were shuffled and represented to the participant. The participant was required to place the 4 odours from left to right into the order of original presentation, i.e. reconstruct the presentation order. The participant was permitted to both smell and re-arrange the position of each odour as many times as required prior to arranging the odours into the order of original presentation. The participant was given a 1-minute rest period prior to presentation of the next sequence.

\section{Results}

In this study a correct response is defined as an item recalled in the same position as that in which it was presented.

\section{$\underline{\text { Serial Position Functions }}$}

We assess the serial position functions for both the non-repeated and repeated sequences (see Figure 1) via a 2-factor $(2 \times 4)$ within-participants ANOVA, where the first factor represents sequence type (repeated sequence versus non-repeated sequences) and the second factor represents serial position (1-4). Consistent with Hebbian learning, recall for the repeated sequence was significantly higher than that for the non-repeated sequences, $F(1,23)=5.70$, $M S E=0.03, p=0.026$, partial $\eta^{2}=0.20$, mean proportion correct $=0.39$ and 0.46 , for nonrepeated and repeated sequences, respectively). The main effect of serial position was significant, $F(3,69)=4.23, M S E=0.08, p=0.008$, partial $\eta^{2}=0.16$, and pairwise comparisons (Bonferroni-corrected, $\alpha=0.008$ ), revealed that proportional correct recall for serial position 4 was significantly higher than that for both serial positions 1 and 2. That the serial position functions were equivalent for both sequence types is evidenced by the null sequence type by serial position interaction, $F<1$. 


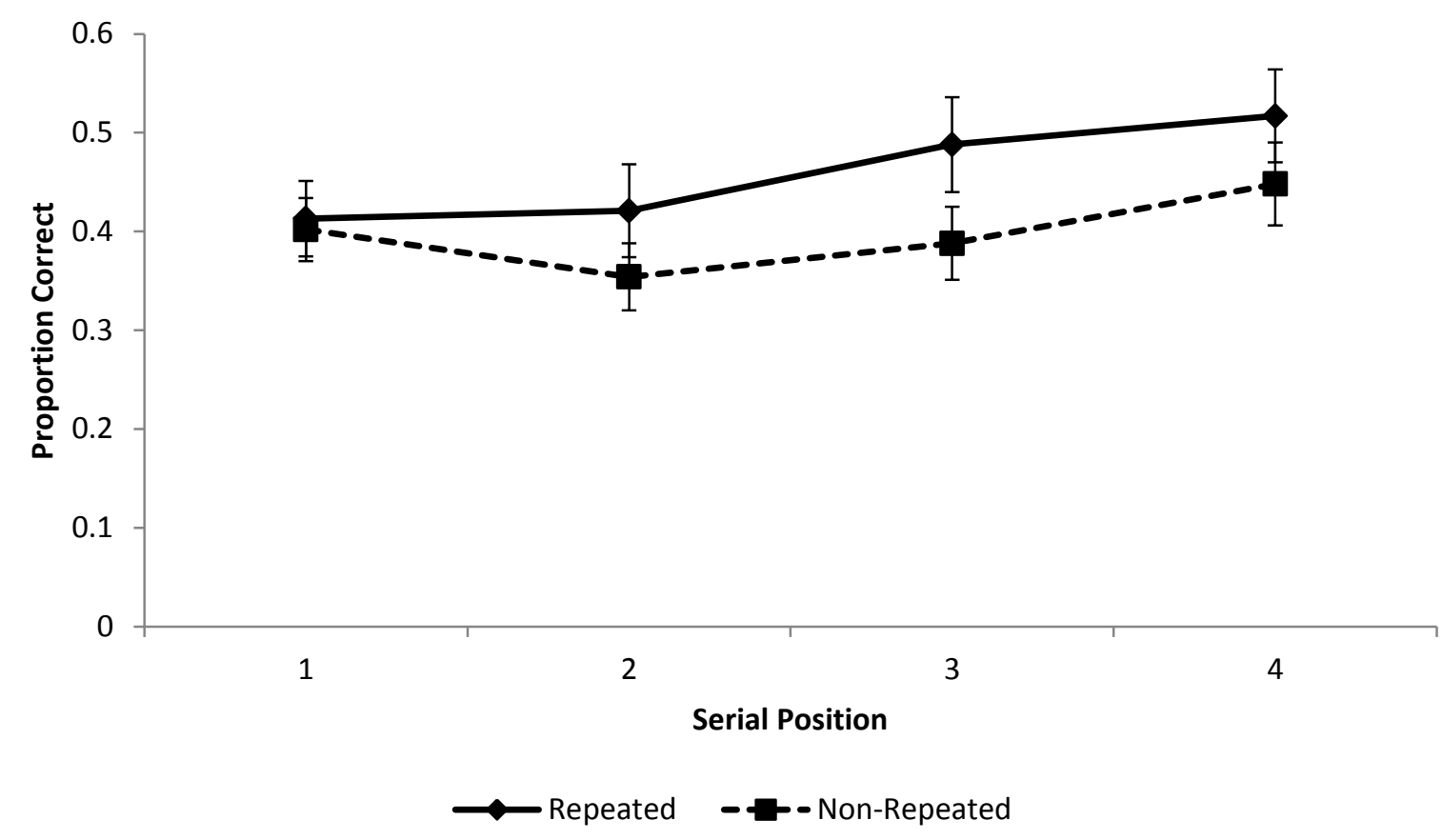

Figure 1: Mean proportion correct for the repeated and non-repeated sequence as a function of serial position (1-4). Error bars denote +/-SEM

\section{Gradients of improvement}

Figure 2 demonstrates that the gradient of improvement is steeper for the repeated sequence relative to the non-repeated sequences. The gradients were compared via a related t-test (2tailed) and, consistent with Hebbian learning, the gradient was significantly steeper for the repeated sequence $(t(23)=2.64, p=0.015$ : regression means $=0.024$ and 0.006 , for the repeated and non-repeated sequences, respectively).

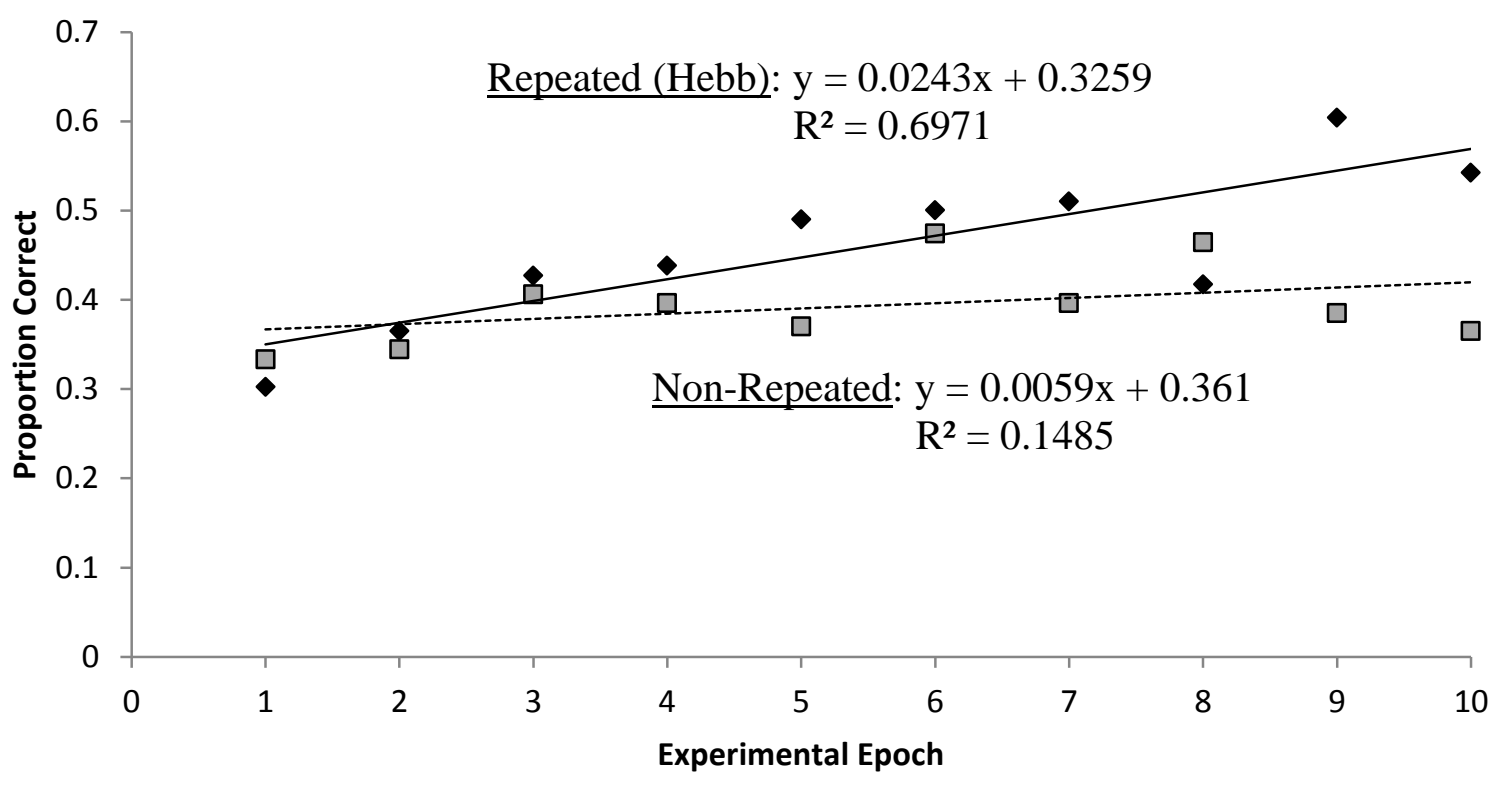

- Repeated $\square \quad$ Non-Repeated Linear (Repeated) Linear (Non-Repeated) 
Figure 2: Mean proportion correct for the repeated and non-repeated sequences as a function of experimental epoch (1-10). Line of best fit depicts the gradient of improvement for each sequence type.

\section{$\underline{\text { Analysis of first and last epoch }}$}

The differences between the non-repeated and repeated sequences for epochs 1 and 10 are shown in Figure 2. A 2-factor (2x2) within-participants ANOVA was conducted where the first factor represents sequence type (non-repeated versus repeated) and the second factor represents experimental epoch (1 versus 10). The main effect of sequence type was nonsignificant, $F(1,23)=2.63, M S E=0.05, p=0.12$, partial $\eta^{2}=0.10$ (mean proportion correct for non-repeated and repeated sequences $=0.35$ and 0.42 , respectively). However, the main effect of experimental epoch was significant, $(F(1,23)=10.07, M S E=0.05, p=0.004$, partial $\eta^{2}=0.35$ ), recall was significant higher for epoch 10 , compared to epoch 1 (mean recall $=0.32$ and 0.45 , for epochs 1 and 10, respectively). Importantly, and consistent with Hebbian learning, the sequence type by experimental epoch interaction was significant, $F(1,23)=6.63$, $M S E=0.04, p=0.017$, partial $\eta^{2}=0.22$. Further analysis (Bonferonni-corrected comparisons, $\alpha=0.025$ ) revealed no difference between the non-repeated and repeated sequences at epoch 1 (mean recall $=0.33$ and 0.30 , respectively). However, at epoch 10 , recall for the repeated sequence compared to the non-repeated sequences was significantly higher (means $=0.54$ and 0.36 , respectively). The interaction reflects the superior learning for the repeated sequence compared to the non-repeated sequences, and is thus consistent with the prediction of Hebbian learning.

\section{Discussion}

The extent to which Hebbian learning is evident for sequences of olfactory stimuli was examined. We describe a novel serial order reconstruction procedure that produced a serial position function comprising recency but not primacy: this function was evident for both the non-repeated and repeated sequences. Importantly, evidence for Hebbian learning was shown via two methods of statistical analysis: (1) the gradient of improvement was significantly steeper for the repeated sequence compared to the non-repeated sequences and, (2) correct proportional recall for the final experimental epoch was significantly higher for the repeated sequence compared to the non-repeated sequences, despite equivalent scores at the first epoch. Taken together these analyses provide compelling evidence for the veridicality of Hebbian learning for olfactory sequences.

This study represents the first empirical demonstration of Hebbian learning for olfactory sequences, and the finding is consistent with the general observation that Hebbian learning is demonstrable for a range of stimulus types, e.g. phonological stimuli (Hebb, 1961), nonverbal visual stimuli (Horton et al., 2008), visuo-spatial stimuli (Couture \& Tremblay, 2006), and audio-spatial stimuli (Lafond et al., 2010). Notwithstanding the fact that this study describes a novel order reconstruction task, it is noteworthy that the gradient of improvement reported here $(0.024)$ is within the range of gradients reported previously, e.g. upright faces 
(0.034: Horton et al., 2008), letters (0.015: Page et al., 2006, Experiment 1) and photographs (0.025 and 0.028: Page et al., 2006, Experiment 2).

That the Hebb repetition effect reported here is not solely a function of verbal recoding is supported by a number of strands of evidence, both theoretical and empirical. Figure 2 shows that recall of the repeated sequence reached approximately $60 \%$ by the final experimental epoch. To the extent that verbal memory span for spoken digits is 7+/-2 (Miller, 1956), we might expect learning for 4 olfactory stimuli to achieve $100 \%$ after 10 trials if the olfactory stimuli were represented as verbal labels, even with articulatory suppression during the ISI (Parmentier, 2012; personal communication). Moreover, if learning of the olfactory stimuli was facilitated via verbal recoding we might further predict a gradient of improvement for the non-repeated trials. This prediction derives from the finding that increased familiarity with odours predicts name-ability (Yeshurun, Dudai, and Sobel, 2008) and nameable odours are characterised by improved memory relative to non-nameable odours (Yeshurun et al., 2008; Jönsson, Møller \& Olsson, 2011). However, a single sample t-test on our data revealed no significant difference between the gradient of improvement for the non-repeated sequences compared to the null hypothesis of $0, t(23)=1.35, p=0.19$.

Evidence against a verbal recoding hypothesis for olfactory sequences can be found in Miles and Hodder (2005, Experiment 6). In a 2AFC recognition task for 5-odour sequences, participants were required to either repeat "the" rapidly and continuously throughout the $5 \mathrm{~s}$ ISI, or remain silent during the ISI. To the extent that participants relied on verbal recoding of the odours to aid later recognition, then we would expect recognition under conditions of articulatory suppression to be impaired compared to recognition under conditions of quiet. In fact, the effect of articulatory suppression was very small (approximately 5\% reduction) and was apparent at only two of the five serial positions. This pattern of data is inconsistent with the proposition that maintenance of odour sequences relies solely on the verbal recoding of the odours. One objection to this conclusion points to the fact that suppression occurred between list items rather than during item presentation. It is possible that suppression is only effective when performed during item presentation as opposed to during the inter-stimulusinterval. And indeed, it is the case that in the current experiment participants were required to suppress during the 3 s. retention interval. The evidence suggests that this locus of of suppression is effective with more typical verbally-based memory tasks. For instance, Toppino and Pisegna (2005) presented participants with lists of consonants to be recalled. Participants performed the task in quiet or were required to suppress either during list presentation or during the $7 \mathrm{~s}$ interval between end of list presentation and the signal to recall. The proportion of recall errors increased dramatically in both suppression conditions $(55 \%$ and $45 \%$ for the through-list and post-list conditions, respectively) compared to the quiet condition (25\%). This demonstrates that articulatory suppression during the rehearsal phase for verbal lists negatively impacts order memory for those lists. By extension, to the extent that participants verbally recoded the olfactory stimuli in the current study, then articulatory suppression during the rehearsal phase only should similarly negatively impact order memory. Taken together, these arguments and evidence make it difficult to defend the 
hypothesis that our results reflect solely the learning of verbal labels assigned to each olfactory stimulus.

The olfactory Hebb repetition effect supports the cross-modal universality of Hebbian learning and is consistent with the view that long-term sequential learning is represented amodally; that is, irrespective of stimulus type (Parmentier, 2011). Indeed, although linked to the acquisition of language (e.g. Mosse \& Jarrold, 2008), long-term sequential learning has been shown to persist despite verbal STM deficits (Mosse \& Jarrold, 2010). Long-term sequence learning may, therefore, be conceptualised as a general, overarching mechanism, that facilitates language acquisition (e.g. deficits in long-term sequence learning has been proposed as a causal factor in dyslexia, Szmalec et al., 2011) but operates cross-modally.

The argument in favour of a universal amodal representational system for sequence order is, however, contradicted by the present serial position function data. The novel reconstruction task produced a function comprising recency in the absence of primacy; a finding that contradicts the characteristic bowed serial position function observed for reconstruction of serial order across a range of other stimulus types (e.g. verbal and non-verbal visual - Ward et al., 2005; spatial - Parmentier \& Jones, 2000). However, whilst the task demands for both our reconstruction procedure and the established serial order reconstruction task appear highly congruent, it is possible that methodological differences between tasks prohibited primacy in the present study. For example, in the present procedure (and in contrast to the conventional reconstruction task), items cannot be re-presented simultaneously at test. The absence of control over the order and duration with which odours were individually evaluated at test may have attenuated primacy via lengthened retention intervals and increased interference. However, it is unclear why this might exhibit deficits specific to primacy and not recency. Application of this novel task to other stimulus types (e.g. non-verbal visual) will provide insight into whether the absence of primacy is an artefact of tasks demands. Nevertheless, a disparate serial position function (and a general absence of primacy) is consistent with our previously published work examining olfactory order memory (Johnson $\&$ Miles, 2009). This is found despite cross-modal serial position equivalence for recognition memory (Johnson \& Miles, 2007; Miles \& Hodder, 2005).

In conclusion, the current data further support the proposal by Page et al. (2006) that "the Hebb repetition effect in ISR extends beyond circumstances in which the phonological loop is used" (p.730), by extending the phenomenon to the chemical senses. The data support the universality of long-term sequential learning and further indicates that this may occur at an abstract, higher order level of cognition. However, the serial position data in the current study, coupled with an earlier report of serial position difference in olfactory order memory (Johnson \& Miles, 2009), suggests that order memory may operate differently for olfactory stimuli and such serial position effects may be independent of the amodal sequential learning mechanism that underpins the Hebb repetition effect. Further work is required to substantiate this potential difference. 


\section{References}

Andrade, J. \& Donaldson, L. (2007). Evidence for an olfactory store in working memory. Psychologia, 50, 76-89.

Couture, M. \& Tremblay, S. (2006). Exploring the characteristics of the visuo-spatial Hebb repetition effect. Memory \& Cognition, 34, 1720-1729.

Guérard, K. \& Tremblay, S. (2008). Revisiting evidence for modularity and functional equivalence across verbal and spatial domains in memory. Journal of Experimental Psychology: Learning, Memory, and Cognition, 34, 556-569.

Hebb, D.O. (1961). Distinctive features of learning in the higher animal. In J.F. Delafresnaye (Ed). Brain mechanisms and learning (p. 37-46). Oxford England: Blackwell.

Horton, N., Hay, D.C. \& Smyth, M.M. (2008). Hebb repetition effects in visual memory: The roles of verbal rehearsal and distinctiveness. Quarterly Journal of Experimental Psychology, 61(12), 1769-1777.

Johnson, A.J. \& Miles, C. (2007). Serial position functions for recognition of olfactory stimuli. Quarterly Journal of Experimental Psychology, 60(10), 1347-1355.

Johnson, A.J. \& Miles, C. (2009). Single-probe serial position recall: evidence of modularity for olfactory, visual and auditory short-term memory. Quarterly Journal of Experimental Psychology, 62(2), 267-275.

Jönsson, F.U., Møller, P. \& Olsson, M.J. (2011). Olfactory working memory: Effects of verbalization on the 2-back task. Memory and Cognition, 39, 1023-1032.

Lafond, D., Tremblay, S. \& Parmentier, F. (2010). The ubiquitous nature of the Hebb repetition effect: Error learning mistaken for the absence of sequence learning. Journal of Experimental Psychology: Learning, Memory, and Cognition, 36(2), 515-522.

Miles, C. \& Hodder, K. (2005). Serial position effects in recognition memory for odours: A re-examination. Memory and Cognition, 33(7), 1303-1314.

Miles, C. \& Jenkins, R. (2000). Recency and suffix effects with immediate recall of olfactory stimuli. Memory, 8, 195-206.

Miller, G.A. (1956). The magic number seven, plus or minus two: Some limits on our capacity for processing information. Psychological Review, 63, 81-93. 
Mosse, E.K. \& Jarrold, C. (2008). Hebb learning, verbal short-term memory, and the acquisition of phonological forms in children. Quarterly Journal of Experimental Psychology, 61, 505-514.

Mosse, E.K. \& Jarrold, C. (2010). Searching for the Hebb effect in Down syndrome: evidence for a dissociation between verbal short-term memory and domain-general learning of serial order. Journal of Intellectual Disability Research, 54(4), 295-307.

Page, M.P.A., Cumming, N., Norris, D., Hitch, G.J. \& McNeil, A.M. (2006). Repetition learning in the immediate serial recall of visual and auditory materials. Journal of Experimental Psychology: Learning memory, and Cognition, 32, 716-733.

Parmentier, F.B.R. (2011). Exploring the determinants of memory for spatial sequences. In A. Vandierendonck \& A. Szmalec (Ed). Spatial Working Memory. Hove, UK: Psychology Press.

Parmentier, F.B.R. \& Jones, D.M. (2000). Functional characteristics of auditory temporalspatial memory: Evidence from serial order errors. Journal of Experimental Psychology: Learning, Memory, and Cognition, 26, 222-238.

Parmentier, F.B.R., Maybery, M.T., Huitson, M. \& Jones, D.M. (2008). The perceptual determinants of repetition learning in auditory space. Journal of Memory and Language, 58, 978-997.

Smith, E.E., Jonides, J. \& Koeppe, R.A. (1996). Dissociating verbal and spatial working memory using PET. Cerebral Cortex, 6, 11-20.

Szmalec, A., Duyck, W., Vandierendonck, A., Barberá Mata, A. \& Page, M.P.A. (2009). The Hebb repetition effect as a laboratory analogue of novel word learning. Quarterly Journal of Experimental Psychology, 62, 435-443.

Szmalec, A., Loncke, M., Duyck, W. \& Page, M.P.A. (2011). Order or disorder? Impaired Hebb learning in dyslexia. Journal of Experimental Psychology: Learning, Memory, and Cognition, 37(5), 1270-1279.

Toppino, T.C. and Pisegna, A. (2005). Articulatory suppression and the irrelevant-speech effect in short-term memory: Does the locus of suppression matter? Psychonomic Bulletin \& Review, 12(2), 374-379.

Ward, G., Avons, S.E. \& Melling, L. (2005). Serial position in curves in short-term memory: Functional equivalence across modalities. Memory, 13(3/4), 308-317.

Yeshurun, Y., Dudai, Y. \& Sobel, N. (2008). Working memory across nostrils. Behavioural Neuroscience, 122(5), 1031-1037. 
Zelano, C., Montag, J., Khan, R. \& Sobel, N. (2009). A specialized odor memory buffer in primary olfactory cortex. PLoS ONE, 4(3), 1-11. 